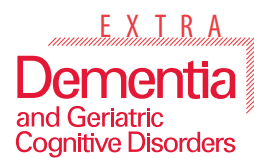

Dement Geriatr Cogn Disord Extra 2013;3:251-262

DOI: 10.1159/000351859

Publisned onine: August 20, 2013 (c) 2013 S. Karger AG, Basel

$1664-5464 / 13 / 0031-0251 \$ 38.00 / 0$ www.karger.com/dee

This is an Open Access article licensed under the terms of the Creative Commons AttributionNonCommercial 3.0 Unported license (CC BY-NC) (www.karger.com/OA-license), applicable to the online version of the article only. Distribution permitted for non-commercial purposes only.

\title{
Clinical Characteristics of C9ORF72-Linked Frontotemporal Lobar Degeneration
}

\author{
Anna-Lotta Kaivorinne ${ }^{a, b}$ Michaela K. Bode ${ }^{c}$ Liisa Paavola ${ }^{a}$ Hannu Tuominen ${ }^{d}$ \\ Mika Kallio $^{\mathrm{e}}$ Alan E. Renton $^{\mathrm{h}}$ Bryan J. Traynor ${ }^{\mathrm{h}}$ Virpi Moilanen $^{\mathrm{a}}$ \\ Anne M. Remes ${ }^{f, g}$

\begin{abstract}
${ }^{a}$ Department of Neurology, Institute of Clinical Medicine, University of Oulu, ${ }^{b}$ Clinical Research Center, Oulu University Hospital, and Departments of ${ }^{\mathrm{c}}$ Diagnostic Radiology, ${ }^{\mathrm{d}}$ Pathology and ${ }^{e}$ Clinical Neurophysiology, Oulu University Hospital, Oulu, ${ }^{\mathrm{f}}$ Department of Neurology, Institute of Clinical Medicine, University of Eastern Finland, and 9Department of Neurology, Kuopio University Hospital, Kuopio, Finland; heuromuscular Diseases Research Unit, Laboratory of Neurogenetics,
\end{abstract} \\ National Institute on Aging, National Institutes of Health, Bethesda, Md., USA
}

\section{Key Words}

Association study · Clinical features · Frontotemporal dementia · Frontotemporal lobar degeneration · Genetics

\begin{abstract}
Background: The most common genetic cause of frontotemporal lobar degeneration (FTLD) and amyotrophic lateral sclerosis (ALS) has been linked to a hexanucleotide repeat expansion in the C9ORF72 gene. The frequency of the C9ORF72 expansion in Finland is among the highest in the world. Methods: We assessed 73 Finnish patients with FTLD in order to examine the clinical characteristics associated with the expanded C9ORF72. Demographic and clinical features were evaluated. As a potential disease modifier, the apolipoprotein E (APOE) genotype was also assessed. Neuropathological analysis was available on 2 expansion carriers and 1 non-carrier. Results: The C9ORF72 expansion was present in 20 of 70 (29\%) probands. Significant associations with the C9ORF72 expansion were observed for concomitant ALS and positive family history of dementia or ALS. Psychoses were detected in both carriers and noncarriers ( 21 vs. $10 \%, p=0.25$ ). The $A P O E \varepsilon 4$ allele did not cluster among expansion carriers. Numerous p62-positive neuronal inclusions were detected in the cerebellar cortex of the 2 expansion carriers. Conclusion: In line with the suggested C9ORF72 core phenotype, we also detected a high frequency of neuropsychiatric symptoms; however, these symptoms seem not be specific to C9ORF72-associated FTLD. FTLD should be considered in cases of middleage-onset psychosis.


Kaivorinne et al.: Clinical Characteristics of C9ORF72-Linked Frontotemporal Lobar Degeneration

\section{Introduction}

Frontotemporal lobar degeneration (FTLD) is a common form of early-onset dementia in patients younger than 65 years [1]. Three major clinical syndromes are recognised: behavioural variant frontotemporal dementia (bvFTD) with predominant behavioural and executive problems; semantic dementia (SD) presenting with loss of semantic memory for words and objects, and progressive non-fluent aphasia (PNFA) presenting with progressive loss of speech and non-fluent speech output [2]. Around 15\% of patients with FTLD also develop amyotrophic lateral sclerosis (ALS; FTLD-ALS) [3].

FTLD and ALS are known to form an overlapping disease spectrum at clinical level [3]. Pathologically, they show underlying TAR DNA-binding protein 43 (TDP-43)-positive inclusions [4]. Recently, the causative gene of the chromosome 9-linked FTLD and ALS has been mapped to a hexanucleotide (GGGGCC) repeat expansion within the gene C9ORF72 [5, 6]. The repeat expansion has been reported to account for $25 \%$ of familial FTLD worldwide [7]; therefore, it seems the most common cause of FTLD. In Finland, the C9ORF72 expansion is exceptionally common in patients with the FTLD-ALS disease spectrum, explaining nearly $50 \%$ of familial FTLD [6]. Irrespective of the population or family history, all repeat expansion carriers seem to share a Finnish founder risk haplotype across the mutation locus [7-9]. The first detailed C9ORF72 genotype-phenotype descriptions have suggested a core phenotype for the C9ORF72-associated FTLD $[10,11]$, albeit a wide variation in clinical phenotype, as well as in demographic characteristics. The aim of our study is to provide a detailed evaluation of the clinical profile of Finnish patients with FTLD caused by the C90RF72 repeat expansion, and to contrast our findings with the suggested core phenotype. Furthermore, the role of the apolipoprotein $\mathrm{E}$ (APOE) genotype as a potential disease modifier was assessed.

\section{Methods}

\section{Study Population}

The cohort consisted of 73 Finnish patients (mean age \pm standard deviation at onset, 58.5 \pm 7.3 years; age range 38-79; 45\% men) diagnosed with FTLD [2] at the Memory Clinic of the Oulu University Hospital, Finland, during the period 1999-2010, and who were clinically followed up until the end of 2011. The mean follow-up period was $3.5 \pm 2.8$ years (range $0.1-11.8)$. The cohort included 46 (63\%) patients with bvFTD, 20 (27\%) with PNFA and 7 $(10 \%)$ with SD. Concomitant ALS was diagnosed in 8 (11\%) patients [12]. FTLD was classified as familial when at least one first-degree relative was reported to suffer from neurodegenerative dementia. Family history was positive for dementia in $26(36 \%)$ cases, for ALS in $3(4 \%)$, and for both in $2(3 \%)$ cases. In those with familial presentation, there were 3 unrelated sibling pairs. Thus, when taking into account only 1 sibling per family, there were altogether 70 apparently unrelated FTLD patients in the cohort. Mutations in the known FTLD genes MAPT, PGRN and $C H M P 2 B$ were previously excluded [13-15]. The cohort was screened for the C9ORF72 repeat expansion as a part of the C9ORF72 mutation discovery study [6] that included 75 patients from our cohort of FTLD patients. The research protocols were approved by the Ethics Committee of the Northern Ostrobothnia Hospital District, following the principles of the Declaration of Helsinki. All patients agreed to participate in the study, and blood samples were obtained after written informed consent from patients and/or their legal representatives.

\section{Neurological Assessment}

Clinical evaluation and follow-up of the patients were carried out by two neurologists. Major behavioural (apathy, disinhibition, aggression, manic behaviour and stereotyped 
Kaivorinne et al.: Clinical Characteristics of C9ORF72-Linked Frontotemporal Lobar Degeneration

behaviour) and psychiatric symptoms (psychiatric depression, psychosis, delusions, hallucinations and paranoia) were recorded, as well as significant language impairment and extrapyramidal signs (tremor, bradykinesia, rigidity and gait impairment). The first Mini-Mental State Examination (MMSE) score at presentation was also present in the patient records. The $A P O E$ genotype was determined for all patients as described previously [16].

\section{Neuropsychological Assessment}

Neuropsychological tests were initially carried out as a part of clinical diagnostics or follow-up, and therefore no uniform neuropsychological test battery was used (see online suppl. methods 1 for a detailed list of tests; for all online supplementary material, see www. karger.com/doi/10.1159/000351859). Impairment in concentration, executive function, verbal knowledge, literacy, verbal memory, non-verbal knowledge, non-verbal memory, spatial orientation, constructive and fine motor skills were each rated on a five-stage scale (scale 0-4), the weakest fifth percentiles (score 0) being those with a poor performance in tests and who could not be assessed psychometrically in that section. A group median was calculated for each sector. The median was selected as the summary statistic as it is less susceptible to the effects of outliers in small amounts of data. Medians were determined for two groups: (1) the analysed C9ORF72 expansion group as a whole ( $\mathrm{n}=20$; mean duration of symptoms at evaluation, $2.6 \pm 2.5$ years) and (2) a subgroup of 9 bvFTD patients in whom the early cognitive profile was available (mean duration of symptoms at evaluation, $2.6 \pm$ 3.3 years). The remaining 11 C9ORF72-positive patients were excluded from the latter analysis because of dementia-staged disease at evaluation and/or prominent language symptoms.

\section{Visual Assessment of Neuroimaging}

The MR images of 12 patients with C9ORF72 expansion were available for retrospective analysis; 1 patient was imaged at $0.23 \mathrm{~T}$ and the rest at $1.5 \mathrm{~T}$. MR images were rated for frontal, temporal and parieto-occipital atrophy on a scale of $0-3$; both sulcal and ventricular dilatation were taken into account [17]. Medial temporal lobe atrophy was assessed using Scheltens' scale (0-4) [18]. Vascular white matter lesions were scored in a manner where periventricular hyperintensity and separate deep white matter hyperintensities are rated separately on a 0-3 rating scale [19]. Assessment of sulcal and ventricular dilatation was mainly based on axial T2-weighted 2-dimensional fluid-attenuated inversion recovery images, and analysis of medial temporal lobe atrophy was based on coronal T1-weighted 3-dimensional images.

Axial CT images of 4 expansion carriers lacking MR images were available for analysis. Frontal, temporal and parieto-occipital atrophy was assessed in the same way as in the MR images. White matter changes were roughly evaluated as absent/mild/moderate/severe. Medial temporal lobe atrophy could not be assessed from the CT scans. Both MR and CT images were also evaluated for cerebellar atrophy. At the time of brain imaging, the mean duration of symptoms was $3.7 \pm 3.7$ years (range $0.5-15.0$ ).

\section{Neurophysiological Assessment}

Routine 21-channel digital EEG was recorded in 7 expansion carriers. Quantitative EEG spectral analysis could be carried out retrospectively in 3 patients. Absolute and relative power was calculated for the delta, theta, alpha and beta frequency bands for each channel. Electroneuromyography (ENMG) examination was carried out in 4 expansion carriers, and in two of them motor-evoked potentials by transcranial magnetic stimulation (TMS-MEP) were also recorded. 


\section{Neuropathological Examination}

Formaldehyde-fixed and paraffin-embedded post-mortem brain and spinal cord tissues were available for analysis in 2 expansion carriers (Nos. 16 and 17) and in 1 non-carrier (No. 23 ) for comparison. Immunohistochemistry on $6-\mu \mathrm{m}$ histological sections of medial frontal gyrus, superior and medial temporal gyrus, hippocampus, brain stem, cerebellum and spinal cord was performed with antibodies against hyperphosphorylated tau, $A \beta, \alpha$-synuclein, p62 and phosphorylated TDP-43 (p-TDP-43) (see online suppl. methods 2 for detailed conditions). The extent of the $\mathrm{p} 62$ and p-TDP-43 pathology was rated for each region semiquantitatively. The pathology of FTLD-TDP was subtyped based on the harmonised classification system [20].

\section{Statistical Analysis}

Differences between two groups were assessed using Student's t test for continuous variables after testing for normal distribution and Pearson's $\chi^{2}$ and Fisher's exact test for categorical variables, as appropriate. Using logistic regression analysis, crude and adjusted odds ratios (ORs) were calculated (the latter adjusted by the variables included in the model) with 95\% confidence intervals (CIs) to evaluate the association of the C9ORF72 repeat expansion with concomitant ALS and family history of dementia or ALS. Survival time was analysed by the Kaplan-Meier method. Moreover, the resulting curves for the expansion carriers and noncarriers were compared with the log-rank test. Survival time was defined as time from symptom onset to death. Patients who were alive at the end of the follow-up period were censored. The significance threshold was 0.05. Analyses were performed using SPSS software version 16.0 (SPSS, Inc., Chicago, Ill., USA).

\section{Results}

\section{Demographic and Clinical Features}

Overall, the C9ORF72 expansion was detected in 22 of 73 patients, of which 13 were familial cases and 9 sporadic. See table 1 for demographic and clinical characteristics of the 22 expansion carriers. The expansion was present in 20 of 70 (29\%) probands. Moreover, $43 \%$ of the probands with a family history of dementia and $21 \%$ of the apparently sporadic cases carried the expansion. To exclude bias in the association analysis, only 1 sibling per family was randomly included in the analyses, i.e. 20 carriers and 50 non-carriers. Detailed results of the association analyses are given in table 2 . In expansion carriers, age at disease onset ranged from 46 to 70 years (mean $58.3 \pm 5.9$ years). Age at disease onset and gender distribution were similar in expansion carriers and non-carriers. Conversely, a significant difference was observed in the family histories of the two groups. A family history of dementia was common in expansion carriers ( $50 \%$ of carriers vs. $26 \%$ of non-carriers, $p=0.05$; crude $\mathrm{OR}=2.8,95 \% \mathrm{CI} 1.0-8.4$ ). In cases of family history of dementia or ALS, the OR for C9ORF72 expansion was 3.5 (95\% CI 1.2-10.3; $\mathrm{p}=0.02)$, and when adjusted for concomitant ALS, the odds were even higher (OR $=5.2,95 \%$ CI $1.5-17.9)$. bvFTD was the most common clinical phenotype among expansion carriers $(n=14,70 \%)$, while there were $6(30 \%)$ patients with PNFA and none with SD. Among non-carriers, the phenotype distributions were 58, 28 and $14 \%$, respectively $(\mathrm{p}=0.35)$. Survival of FTLD patients without concomitant ALS did not differ between carriers and non-carriers $(p=0.92)$. Interestingly, we detected 1 expansion carrier (No.14) with an extremely long disease course of nearly two decades.

Concomitant ALS was diagnosed in 6 (30\%) expansion carriers versus 2 (4\%) noncarriers $(\mathrm{p}=0.005)$ and was associated with increased odds of having the expansion (crude $\mathrm{OR}=10.3,95 \% \mathrm{CI} 1.9-56.7)$. The association was even more pronounced when adjusted for 
Kaivorinne et al.: Clinical Characteristics of C9ORF72-Linked Frontotemporal Lobar Degeneration

Table 1. Summary of demographic and clinical characteristics of patients with C9ORF72 repeat expansion

\begin{tabular}{|c|c|c|c|c|c|c|c|c|c|c|}
\hline \multirow{2}{*}{$\begin{array}{l}\text { Patient } \\
\text { No. }\end{array}$} & \multirow[t]{2}{*}{ Sex } & \multirow{2}{*}{$\begin{array}{l}\text { Clinical } \\
\text { diagnosis }\end{array}$} & \multirow{2}{*}{$\begin{array}{l}\text { FTLD } \\
\text { subtype }\end{array}$} & \multirow{2}{*}{$\begin{array}{l}\text { ALS } \\
\text { onset }\end{array}$} & \multicolumn{2}{|c|}{ Family history } & \multicolumn{2}{|c|}{ Age at onset, years } & \multirow[t]{2}{*}{ Age, years } & \multirow{2}{*}{$\begin{array}{l}\text { Duration, } \\
\text { years }\end{array}$} \\
\hline & & & & & FTLD & ALS & FTLD & ALS & & \\
\hline 1 & $\mathrm{~F}$ & FTLD & bvFTD & - & No & No & 58 & - & 64 (current) & $>6$ \\
\hline 2 & M & FTLD & bvFTD & - & Yes & No & 57 & - & 62 (current) & $>5$ \\
\hline 3 & $\mathrm{~F}$ & FTLD & bvFTD & - & Yes & No & 64 & - & 75 (current) & $>11$ \\
\hline $4^{a}$ & $\mathrm{~F}$ & FTLD & bvFTD & - & Yes & Yes & 60 & - & 72 (current) & $>12$ \\
\hline 5 & M & FTLD & bvFTD & - & Yes & No & 54 & - & 66 (current) & $>12$ \\
\hline $6^{b}$ & $\mathrm{~F}$ & FTLD & bvFTD & - & Yes & No & 47 & - & 53 (current) & $>6$ \\
\hline 7 & $\mathrm{~F}$ & FTLD & bvFTD & - & No & No & 51 & - & 54 (current) & $>3$ \\
\hline 8 & M & FTLD & bvFTD & - & Yes & No & 55 & - & 60 (current) & $>5$ \\
\hline 9 & M & FTLD & bvFTD & - & Yes & No & 54 & - & 60 (current) & $>6$ \\
\hline 10 & M & FTLD & bvFTD & - & Yes & No & 46 & - & 49 (current) & $>3$ \\
\hline 11 & $\mathrm{~F}$ & FTLD & bvFTD & - & Yes & No & 59 & - & 63 (current) & $>4$ \\
\hline 12 & M & FTLD & bvFTD & - & Yes & Yes & 64 & - & 68 (current) & $>4$ \\
\hline 13 & $\mathrm{~F}$ & FTLD & PNFA & - & No & No & 60 & - & 71 (at death) & 11 \\
\hline 14 & M & FTLD & PNFA & - & No & No & 51 & - & 70 (current) & $>19$ \\
\hline 15 & M & FTLD & PNFA & - & No & No & 64 & - & 71 (at death) & 7 \\
\hline 16 & $\mathrm{~F}$ & FTLD & PNFA & - & Yes & No & 63 & - & 72 (at death) & 9 \\
\hline 17 & $\mathrm{~F}$ & FTLD-ALS & bvFTD & bulbar & No & No & 59 & 60 & 62 (at death) & 3 \\
\hline 18 & $\mathrm{~F}$ & FTLD-ALS & PNFA & bulbar & No & Yes & 54 & 55 & 58 (at death) & 4 \\
\hline 19 & M & FTLD-ALS & PNFA & bulbar & Yes & No & 70 & 70 & 71 (at death) & 1 \\
\hline 20 & M & FTLD-ALS & bvFTD & bulbar & No & No & 57 & 57 & 60 (at death) & 3 \\
\hline 21 & $\mathrm{~F}$ & FTLD-ALS & bvFTD & spinal & No & No & 60 & 60 & 63 (at death) & 3 \\
\hline 22 & $\mathrm{~F}$ & FTLD-ALS & bvFTD & bulbar & No & No & 66 & 66 & 69 (at death) & 3 \\
\hline
\end{tabular}

${ }^{a}$ The sibling is patient No.12, patient No. 4 was excluded from the association analysis. ${ }^{\mathrm{b}}$ The sibling is patient No. 5 , patient No. 6 was excluded from the association analysis.

family history of dementia or ALS (OR $=16.4,95 \%$ CI 2.6-104.1). ALS was associated with bvFTD in 4 expansion carriers and with PNFA in 2. The symptoms of ALS were present or developed within 1 year after the first FTLD symptoms. The site of ALS onset was bulbar in 5 (83\%) expansion carriers and spinal in $1(17 \%)$. The only 2 non-carriers with concomitant ALS had spinal onset of the disease.

Detailed clinical data were available for 68 probands (19 carriers and 49 non-carriers). Among the first symptoms, relative-reported memory complaints were present in $13(68 \%)$ carriers and in $36(74 \%)$ non-carriers ( $\mathrm{p}=0.68)$. At the time of referral, the mean MMSE score was similar in both groups (table 2). During the disease course, mild extrapyramidal symptoms were present in $5(26 \%)$ carriers and $10(20 \%)$ non-carriers $(p=0.75)$. Prominent behavioural symptoms were common in both groups (84 vs. 88\%; $p=0.70$ ). Apathy (53 vs. $61 \%$ ), stereotyped behaviour ( 42 vs. $39 \%$ ) and disinhibition (37 vs. $12 \%$ ) were the most common behavioural symptoms. Problems in speech and language were also often encountered (84 vs. $69 \%$; $p=0.21)$. There was a history of major psychosis in $4(21 \%)$ expansion carriers. Psychosis was their leading symptom, and bvFTD was diagnosed within 1-5 years after psychosis. Delusions and auditory hallucinations were the most common psychiatric symptoms among expansion carriers; none had visual hallucinations. Psychosis was also diagnosed in $5(10 \%)$ non-carriers $(\mathrm{p}=0.25)$. Preceding the diagnosis of FTLD, major depression was suspected in both groups ( $16 \%$ of carriers vs. $20 \%$ of non-carriers; $p=0.99$ ). None of the patients with psychosis developed concomitant ALS. 
Kaivorinne et al.: Clinical Characteristics of C9ORF72-Linked Frontotemporal Lobar Degeneration

Table 2. Clinical comparison between groups of FTLD patients with and without expanded C9ORF72

\begin{tabular}{|c|c|c|c|c|c|}
\hline Variable & $\begin{array}{l}\text { Non-expanded } \\
\text { C9ORF72 } \\
(\mathrm{n}=50)\end{array}$ & $\begin{array}{l}\text { Expanded } \\
C 9 O R F 72 \\
(\mathrm{n}=20)\end{array}$ & $\mathrm{p}$ value & $\begin{array}{l}\text { Crude OR } \\
(95 \% \mathrm{CI})\end{array}$ & $\begin{array}{l}\text { Adjusted OR } \\
(95 \% \mathrm{CI})\end{array}$ \\
\hline Age at onset, years & $58.8 \pm 7.9$ & $58.3 \pm 5.9$ & 0.81 & & \\
\hline Range, years & $38-79$ & $46-70$ & - & & \\
\hline Survival, years ${ }^{\mathrm{a}}$ & $13.8 \pm 0.8$ & $14.0 \pm 2.1$ & 0.92 & & \\
\hline Male/female (male \%) & $22 / 28(44)$ & $10 / 10(50)$ & 0.65 & & \\
\hline \multicolumn{6}{|l|}{ FTLD phenotype } \\
\hline Behavioural variant & $29(58)$ & $14(70)$ & 0.35 & & \\
\hline Language variant ${ }^{\mathrm{b}}$ & $21(42)$ & $6(30)$ & - & & \\
\hline Concomitant ALS & $2(4)$ & $6(30)$ & $0.005^{*}$ & $10.3(1.9-56.7)$ & $16.4(2.6-104.1)^{\mathrm{d}}$ \\
\hline \multicolumn{6}{|l|}{ Positive family history } \\
\hline Dementia or ALS & $13(26)$ & $11(55)$ & $0.02^{*}$ & $3.5(1.2-10.3)$ & $5.2(1.5-17.9)^{\mathrm{d}}$ \\
\hline Dementia & $13(26)$ & $10(50)$ & 0.05 & $2.8(1.0-8.4)$ & \\
\hline ALS & $0(0)$ & $2(10)$ & NA & & \\
\hline MMSE at referral $^{\mathrm{C}}$ & $22.9 \pm 4.6$ & $24.3 \pm 4.8$ & 0.31 & & \\
\hline
\end{tabular}

Values are numbers (\%) unless otherwise specified. Continuous variables are shown as mean $\pm \mathrm{SD}$. NA = Not applicable. Statistically significant: ${ }^{*} \mathrm{p}<0.05$.

${ }^{a}$ Survival from symptom onset to death. Patients with concomitant ALS were excluded. Data included censored data (11 for C9ORF72 expansion carriers and 33 for non-carriers). ${ }^{\mathrm{b}} \mathrm{PNFA}+\mathrm{SD} .{ }^{\mathrm{c}} \mathrm{MMSE}$ score $0-$ 30. Data available for 16 expansion carriers and 46 non-carriers. ${ }^{\mathrm{d}}$ Adjusted for concomitant ALS and positive family history of dementia or ALS.

\section{Cognitive Features}

Analysis of the early cognitive profile revealed that severe executive dysfunction was already present at early stages of the disease (scale 1/4) (fig. 1b). Constructive skills, nonverbal knowledge and memory were also substantially affected (2/4). When the C9ORF72 group was analysed as a whole, there was a substantial fall in all neuropsychological domains except literacy (fig. 1c). Concentration declined rapidly in all cases (0/4) and was the most severely affected domain besides executive functions (1/4). Deficits in fine motor skills, nonverbal knowledge, non-verbal memory as well as verbal knowledge were also salient $(2 / 4)$. Verbal memory was slightly affected (3/4).

\section{Neuroimaging Features}

The results of visual analyses of brain MR and CT images of the C9ORF72 expansion carriers are summarised in online supplementary table 1 . In the analyses, no uniform type of atrophy was detected. However, trends towards two different types of atrophy were observed: one with prevalent frontotemporal cortical atrophy often associated with frontal horn dilatation (fig. 2a, b), and the other with more generalised and prominent ventricular dilation (fig. 2c, d). Cortical atrophy also extended occasionally to the parieto-occipital region (fig. 2g, h). Moderate-to-severe ventricular enlargement was detected in 10 of 16 cases, it was mainly frontal and showed various combinations. Atrophy was mainly symmetrical. Medial temporal lobe atrophy varied from grade 0 to 3 . Signs of minor cerebellar degeneration were only rarely found. Mild changes were detected in the white matter. 
Fig. 1. Neuropsychological features of the patients with C9ORF72 repeat expansion. a Neuropsychological profile of a healthy individual. b Early cognitive profile in a subset of C9ORF72 expansion carriers with bvFTD $(n=9)$. c Cognitive profile of all FTLD cases with expanded C9ORF72 ( $\mathrm{n}=20$ ). The results are displayed as modified McFie's target diagrams [35]. Each sector of the diagram shows a particular cognitive domain. Impairment of a domain was rated in five stages (0-4). In the patient diagrams, the level of function is presented with a group median value.

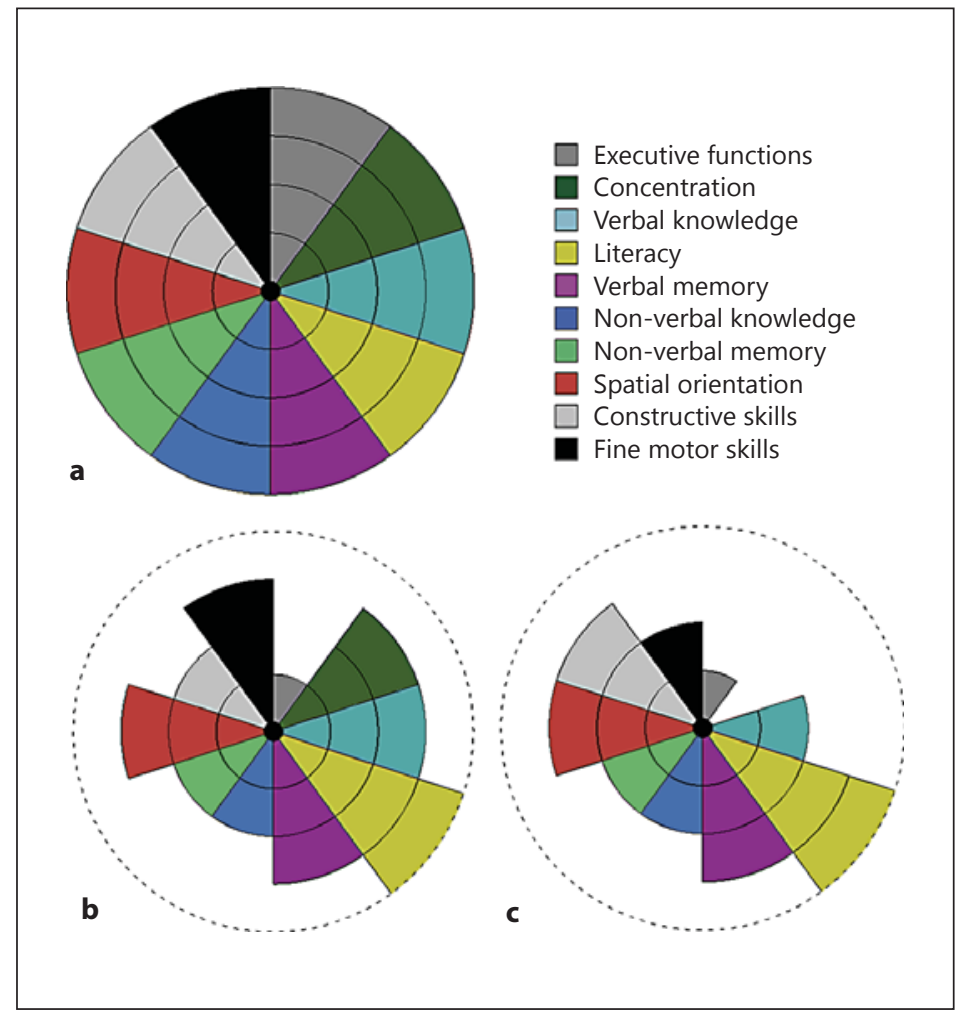

\section{Neurophysiological Features}

Two patients with repeat expansion developed epileptic seizures during the disease course. EEG findings were abnormal in 4 of 7 patients with C9ORF72 expansion. Generalised slowing of background activity was detected in 2 patients. Two patients showed intermittent delta-theta activity in the left temporal region. Quantitative EEG spectral analysis of 3 patients did not show any further abnormal activity. In 2 patients, the ENMG findings revealed clinically definite ALS [12]. In the remaining 2 patients, abnormal bulbar ENMG and TMS-MEP confirmed the diagnosis.

\section{APOE Allele}

There was no statistically significant difference in the $A P O E \varepsilon 4$ allele frequency between the expansion carriers and non-carriers ( 20 vs. $18 \% ; \mathrm{p}=0.78$ ), nor were there significant differences in the $A P O E \varepsilon 2$ allele frequency (10 vs. $6 \%$; $\mathrm{p}=0.62$ ). In the $C 9 O R F 72$ expansion group, $A P O E \& 4$ carriers were found both in patients with and without ALS (67 vs. $21 \%$ ). Among expansion carriers, mean age at onset was $61.1 \pm 5.7$ years in APOE $\varepsilon 4$ carriers and $56.8 \pm 5.6$ years in $A P O E$ \& 4 non-carriers $(\mathrm{p}=0.11$ ).

\section{Neuropathology}

Neuropathological findings are summarised in table 3. The PNFA patient (No. 16) showed an extremely atrophic neocortex and hippocampus on routine staining. One bvFTD-ALS patient (No. 17) had neuronal loss in the frontal cortex and also in the temporal neocortex and hippocampus to a lesser extent, while the other (No. 23) showed no atrophic changes in routine staining. Macroscopically, there was no pronounced cerebellar atrophy in any of these patients. However, the PNFA patient showed cerebellar gliosis and scarce Purkinje cells. The 2 cases with concomitant ALS had degeneration of hypoglossal nuclear neurons and 
Kaivorinne et al.: Clinical Characteristics of C9ORF72-Linked Frontotemporal Lobar Degeneration

Fig. 2. Examples of different patterns of atrophy in patients with C9ORF72 repeat expansion. a, b Prominent frontal atrophy in patient No. 11, 2 years after symptom onset. c Relatively mild central atrophy in patient No. 20, 1 year after symptom onset. d Severe central atrophy with less prominent cortical atrophy in patient No. 15, 4 years after symptom onset. e-g Mild cortical atrophy in patients Nos. 14 (e, f) and $6(\mathbf{g})$. At the time of imaging, patient No. 14 had suffered from symptoms of FTLD for 15 years. g, h Parietal cortical atrophy with small infarction on the left in patient No. 6, 3.5 years after symptom onset. Transaxial T2-weighted fluid-attenuated inversion recovery MR image (a, c, e, g). Coronal T1weighted 3-dimensional MR image $(\mathbf{b}, \mathbf{f}, \mathbf{h})$. Axial CT image (d).

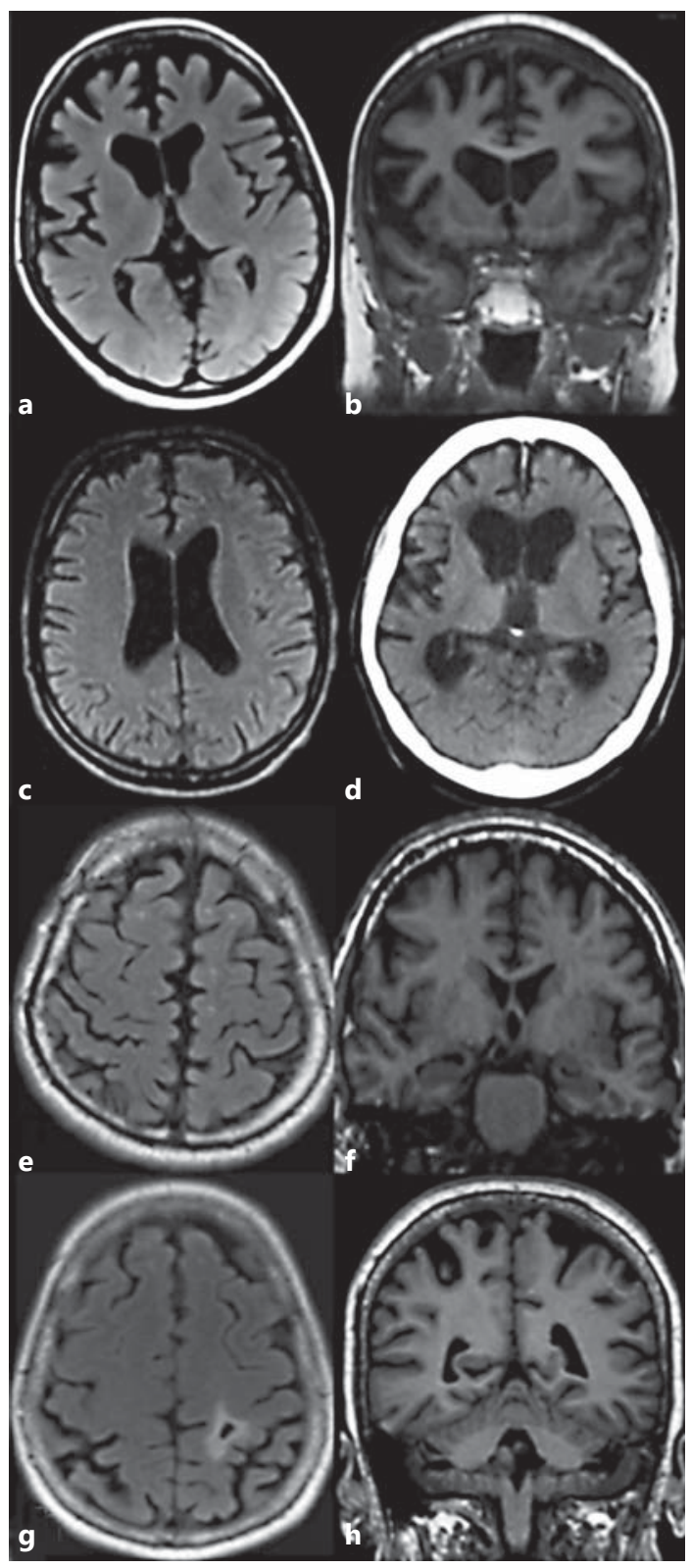

spinal anterior horn cells. Occasional tau-positive neurons and neuropil threads in the hippocampus were seen in the expansion carrier (No.17).

Immunohistochemistry for p-TDP-43 and p62 labelled neuronal cytoplasmic inclusions (NCIs), dystrophic neurites and occasional glial cytoplasmic inclusions in a wide range of brain regions. Of note, in the neocortex, p62 staining was less intense than p-TDP-43 staining, whereas in the hippocampus the stainings were of similar intensity. The patient with PNFA (No. 16) had FTLD-TDP type B pathology, whereas one of the patients with bvFTD-ALS (No. 17) had type A. The most specific findings as regards the expansion carriers were p62-positive, p-TDP-43-negative NCIs in cerebellar molecular and granular layers. In the cases with concomitant ALS, a p-TDP-43-positive lower motor neuron pathology was detected, yet on a small scale for the expansion carrier. Pyramidal tract pathology was also detected in the patient without clinical ALS. 
Kaivorinne et al.: Clinical Characteristics of C9ORF72-Linked Frontotemporal Lobar Degeneration

Table 3. Neuropathological findings, including detailed immunohistochemical results (p62 and p-TDP-43), for 2 cases (Nos. 16 and 17) with and 1 case (No. 23) without C9ORF72 expansion

\begin{tabular}{|c|c|c|c|}
\hline & \multicolumn{3}{|l|}{ Patient No. } \\
\hline & 16 & 17 & 23 \\
\hline Phenotype & PNFA & bvFTD-ALS & bvFTD-ALS \\
\hline Brain weight, g & 733 & 1,184 & 1,217 \\
\hline Gross atrophy & generalised & no atrophy & frontotemporal \\
\hline \multicolumn{4}{|l|}{$I H C p 62 / p-T D P-43^{a}$} \\
\hline \multicolumn{4}{|l|}{ Frontal cortex } \\
\hline $\mathrm{NCI}$ & $+/+$ & $++/+++$ & $+/+++$ \\
\hline DN & $+/++$ & $-/+++$ & $(+) /++$ \\
\hline \multicolumn{4}{|l|}{ Temporal cortex } \\
\hline NCI & $+/+$ & $(+) /++$ & $(+) /++$ \\
\hline DN & $+/++$ & $-/+++$ & $-/++$ \\
\hline \multicolumn{4}{|l|}{ Hippocampus } \\
\hline Pyramidal NCI & $(+) /+$ & $+/+$ & $-/(+)$ \\
\hline Granular NCI & $+++/++$ & $+++/+++$ & $++/++$ \\
\hline $\mathrm{DN}$ & $-/-$ & $-/-$ & $-/-$ \\
\hline \multicolumn{4}{|l|}{ Cerebellum } \\
\hline Purkinje cells & $-/-$ & $-/-$ & $-/-$ \\
\hline Dentate neurons & $-/-$ & $-/-$ & $-/-$ \\
\hline Molecular layer NCI & $++/-$ & $+/-$ & $-/-$ \\
\hline Granular layer NCI & $+++/-$ & $++/-$ & $-/-$ \\
\hline DN & $-/-$ & $-/-$ & $-/-$ \\
\hline GCI & $-/-$ & $-/(+)$ & $-/(+)$ \\
\hline \multicolumn{4}{|l|}{ Brain stem } \\
\hline ON pyramidal NCI & $+/++$ & $-/+$ & $++/++$ \\
\hline HN/VN pyramidal NCI & $(+) /-$ & $-/-$ & $(+) /+++$ \\
\hline DN & $-/+$ & $-/-$ & $-1-$ \\
\hline GCI & $-/-$ & $(+) /(+)$ & $-/-$ \\
\hline \multicolumn{4}{|l|}{ Spinal cord anterior horn } \\
\hline $\mathrm{NCI}$ & $-/-$ & $-/+$ & $+/++$ \\
\hline DN & $-/-$ & $-/-$ & $-/-$ \\
\hline GCI & $-1-$ & $(+) /(+)$ & $+/+$ \\
\hline FTLD-TDP type & $\mathrm{B}$ & $\mathrm{A}$ & \\
\hline
\end{tabular}

DN = Dystrophic neurites; GCI = glial cytoplasmic inclusions; $\mathrm{HN}=$ hypoglossal nucleus; $\mathrm{IHC}=$ immunohistochemistry; $\mathrm{ON}$ = olivary nucleus; $\mathrm{VN}$ = vagal nucleus. ${ }^{\mathrm{a}}$ IHC was scored using a semiquantitative grading system: - negative; (+) occasional; + few; ++ moderate; +++ numerous. ${ }^{\text {b }}$ Subtyped according to the harmonised classification system for FTLD-TDP pathology (type A-D) [20].

\section{Discussion}

The C9ORF72 repeat expansion explains nearly $50 \%$ of familial and $30 \%$ of all FTLD cases in the Finnish population. The frequency of the C9ORF72 expansion in Finland is among the highest in the world, and the sporadic form of the disease is also relatively common, as the mutation has been detected in $19 \%$ of cases of sporadic FTLD $[6,7]$. The high frequency of the C9ORF72 expansion is likely to reflect the Finnish population history. Haplotype analysis of C9ORF72 expansion carriers has suggested that all patients with the expansion, both familial and sporadic cases, share a common Finnish founder risk haplotype [7-9].

bvFTD was the most common phenotype in patients with repeat expansion. Moreover, one third of the patients presented with PNFA, the frequency being similar in non-carriers. 
SD was absent in the C9ORF72 expansion group. In previous reports, PNFA has been rare or absent in patients with C9ORF72 expansion [10,11,21]. A variety of salient behavioural symptoms as well as major psychiatric symptoms were found in both groups. Among our expansion carriers, major psychosis was diagnosed in $21 \%$ of the patients, and it is noteworthy that psychosis may be the first symptom in a C $90 R F 72$ carrier, even years before other symptoms occur. Both mild and florid psychotic symptoms have been reported to be common in C9ORF72 expansion carriers [10,21,22]. However, it is notable that patients without repeat expansion also presented with major psychosis with a frequency not statistically different from expansion cases.

As expected, ALS was more common in our FTLD patients with C9ORF72 expansion than in those without $[5,6]$. The bulbar-onset type of ALS seems to be associated with the expansion [21-25], and a similar trend was also detected in our study. The repeat expansion did not significantly affect survival in FTLD patients without ALS. Nonetheless, the number of deceased patients was low in our cohort. To date, C9ORF72 expansion has not been associated with shorter disease duration in pure FTLD cases [24], in contrast to C9ORF72-linked ALS [23, $24,26,27]$.

The MRI findings did not reveal any uniform profile of atrophy in patients with repeat expansion. However, two distinct types of atrophy could be extracted: one with prevalent frontal cortical atrophy and the other with more generalised ventricular dilation. A similar ventricular enlargement has been reported in a longitudinal MRI analysis of expansion carriers $[10,11,28]$. In general, atrophy was symmetrical, which is consistent with previous reports $[10,11,29,30]$. In voxel-based and volumetric MRI analyses, C9ORF72 expansion groups have been characterized by widespread and varying patterns of atrophy, especially in the frontal and also in the temporal lobes, spreading even further to the parietal and occipital lobes and to the cerebellum $[11,28,30]$. In our cohort, parietal atrophy was also evident in some cases, whereas distinct cerebellar atrophy was not detected. While we were not able to detect cerebellar atrophy in the visual MRI analysis, the detection of p62-positive cerebellar inclusions supports the role of the cerebellum as an important region of C9ORF72-associated pathology [31]. However, in this study, there was no evidence of clinical cerebellar motor symptoms. The role of cerebellar atrophy and p62-positive inclusions is still unknown in C9ORF72 expansion carriers.

Phenotypic variation is wide in C9ORF72 expansion carriers [29], and the possible disease-modifying factors are still unknown. APOE $\varepsilon 4$ has been shown to enhance brain atrophy in disease-specific regions in FTLD patients [32]. More recently, in an FTLD-ALS sibling pair linked to C9ORF72 expansion, the APOE $\varepsilon 4$ homozygous patient has been shown to have more severe cognitive behavioural symptoms and neuropathology than the patient with $\varepsilon 3$ homozygosity [33]. In our study, the $\varepsilon 4$ allele did not seem to contribute to the phenotype. Nevertheless, the role of $A P O E$ as a potential disease modifier in C9ORF72-associated FTLD-ALS warrants further studies with a larger patient population and correlation to neuropathological findings. Anticipation is a phenomenon associated with repeat expansion disorders. Furthermore, the size of the expansion correlates with the age of onset and disease severity to some extent. In reports on expanded C9ORF72, a trend towards genetic anticipation has been suspected $[10,22,29]$. However, as the current repeat-primed PCR method for the detection of C9ORF72 expansion does not allow measurement of the repeat size, the phenomenon cannot be reliably studied.

The present study has some limitations. We could have added more value to genotypephenotype correlations if each patient in the cohort had undergone a similar battery of investigations and the symptoms of the patients had been collected using a standard structured form. We might have missed some minor neuropsychiatric symptoms, as no standardised measurement of neuropsychiatric symptoms was used throughout the cohort. In the neuropsychological assessment, the heterogeneity of the patient cohort and the small number of 
patients did not allow the use of sample mean as a characteristic statistic. Furthermore, cognitive domains were used instead of reporting single test results, as no uniform neuropsychological test battery was employed. Lastly, visual analysis of brain MR images did not allow further conclusions to be drawn from the imaging results. Nevertheless, the results from the volumetric analyses are also difficult to translate into routine clinical practice [34], and thus our visual analyses are of value for clinical practice.

In conclusion, a wide range of demographic features as well as phenotype was found in our cohort of C9ORF72 expansion carriers. A distinctive core phenotype for C9ORF72-positive FTLD has been suggested $[10,11]$. Our results are mostly in line with this core phenotype showing a high frequency of neuropsychiatric symptoms. Yet, in our cohort, neuropsychiatric symptoms were not specific to C9ORF72-linked FTLD. The psychotic presentations, both in C9ORF72 expansion carriers and non-carriers, suggest that FTLD should be considered in cases of middle-age-onset psychosis.

\section{Acknowledgements}

We thank all patients and their families for participating in this study. Ms. Anja Heikkinen and Ms. Pirjo Keränen are acknowledged for their excellent technical assistance. This work was supported in part by the Finnish Medical Foundation, the Health Care Foundation of Northern Finland, EVO grants from the Oulu University Hospital, and the Intramural Research Programs of the NIH, National Institute on Aging (Z01-AG000949-02). B.J.T. was supported by the ALS Association, AriSLA, Packard Center for ALS research, FIGC, Microsoft Research, and the Myasthenia Gravis Foundation. A.M.R. was supported by the Finnish Medical Society Duodecim and the Health Care Foundation of Northern Finland.

\section{Disclosure Statement}

B.J.T. has a patent pending on the diagnostic and therapeutic uses based on the discovery of the hexanucleotide repeat expansion in C9ORF72. The remaining authors disclose no conflicts of interest.

\section{References}

1 Ratnavalli E, Brayne C, Dawson K, Hodges JR: The prevalence of frontotemporal dementia. Neurology 2002; 58:1615-1621.

-2 Neary D, Snowden JS, Gustafson L, Passant U, Stuss D, Black S, Freedman M, Kertesz A, Robert PH, Albert M, Boone K, Miller BL, Cummings J, Benson DF: Frontotemporal lobar degeneration: a consensus on clinical diagnostic criteria. Neurology 1998;51:1546-1554.

$>3$ Lomen-Hoerth C, Anderson T, Miller B: The overlap of amyotrophic lateral sclerosis and frontotemporal dementia. Neurology 2002;59:1077-1079.

4 Neumann M, Sampathu DM, Kwong LK, et al: Ubiquitinated TDP-43 in frontotemporal lobar degeneration and amyotrophic lateral sclerosis. Science 2006;314:130-133.

-5 DeJesus-Hernandez M, Mackenzie IR, Boeve BF, et al: Expanded GGGGCC hexanucleotide repeat in noncoding region of C90RF72 causes chromosome 9p-linked FTD and ALS. Neuron 2011;72:245-256.

6 Renton AE, Majounie E, Waite A, et al: A hexanucleotide repeat expansion in C90RF72 is the cause of chromosome 9p21-linked ALS-FTD. Neuron 2011;72:257-268.

7 Majounie E, Renton AE, Mok K, et al: Frequency of the C9orf72 hexanucleotide repeat expansion in patients with amyotrophic lateral sclerosis and frontotemporal dementia: a cross-sectional study. Lancet Neurol 2012; 11:323-330.

8 Laksovirta H, Peuralinna T, Schymick JC, et al: Chromosome 9p21 in amyotrophic lateral sclerosis in Finland: a genome-wide association study. Lancet Neurol 2010;9:978-985. 


\begin{tabular}{l|l}
\hline DOI: $10.1159 / 000351859$ & $\begin{array}{l}\text { C) } 2013 \text { S. Karger AG, Basel } \\
\text { www.karger.com/dee }\end{array}$ \\
\hline
\end{tabular}

Kaivorinne et al.: Clinical Characteristics of C9ORF72-Linked Frontotemporal Lobar Degeneration

-9 Mok K, Traynor BJ, Schymick J, et al: The chromosome 9 ALS and FTD locus is probably derived from a single founder. Neurobiol Aging 2012;33:209.e3-209.e8.

-10 Boeve BF, Boylan KB, Graff-Radford NR, et al: Characterization of frontotemporal dementia and/or amyotrophic lateral sclerosis associated with the GGGGCC repeat expansion in C90RF72. Brain 2012;135:765-783.

11 Mahoney CJ, Beck J, Rohrer JD, et al: Frontotemporal dementia with the C90RF72 hexanucleotide repeat expansion: clinical, neuroanatomical and neuropathological features. Brain 2012;135:736-750.

-12 Brooks BR, Miller RG, Swash M, Munsat TL, World Federation of Neurology Research Group on Motor Neuron Diseases: El Escorial revisited: revised criteria for the diagnosis of amyotrophic lateral sclerosis. Amyotroph Lateral Scler Other Motor Neuron Disord 2000;1:293-299.

13 Kaivorinne AL, Krüger J, Kuivaniemi K, Tuominen H, Moilanen V, Majamaa K, Remes AM: Role of MAPT mutations and haplotype in frontotemporal lobar degeneration in Northern Finland. BMC Neurol 2008;8:48.

14 Krüger J, Kaivorinne AL, Udd B, Majamaa K, Remes AM: Low prevalence of progranulin mutations in Finnish patients with frontotemporal lobar degeneration. Eur J Neurol 2009;16:27-30.

-15 Kaivorinne AL, Krüger J, Udd B, Majamaa K, Remes AM: Mutations in CHMP2B are not a cause of frontotemporal lobar degeneration in Finnish patients. Eur J Neurol 2010;17:1393-1395.

16 Wenham PR, Price WH, Blandell G: Apolipoprotein E genotyping by one-stage PCR. Lancet 1991;337:11581159.

17 Pasquier F, Leys D, Weerts JG, Mounier-Vehier F, Barkhof F, Scheltens P: Inter- and intraobserver reproducibility of cerebral atrophy assessment on MRI scans with hemispheric infarcts. Eur Neurol 1996;36:268-272.

18 Scheltens P, Leys D, Barkhof F, Huglo D, Weinstein HC, Vermersch P, Kuiper M, Steinling M, Wolters EC, Valk J: Atrophy of medial temporal lobes on MRI in 'probable' Alzheimer's disease and normal ageing: diagnostic value and neuropsychological correlates. J Neurol Neurosurg Psychiatry 1992;55:967-972.

19 Fazekas F, Chawluk JB, Alavi A, Hurtig HI, Zimmerman RA: MR signal abnormalities at 1.5 T in Alzheimer's dementia and normal aging. AJR Am J Roentgenol 1987;149:351-356.

20 Mackenzie IR, Neumann M, Baborie A, Sampathu DM, Du Plessis D, Jaros E, Perry RH, Trojanowski JQ, Mann DM, Lee VM: A harmonized classification system for FTLD-TDP pathology. Acta Neuropathol 2011;122:111113.

21 Snowden JS, Rollinson S, Thompson JC, et al: Distinct clinical and pathological characteristics of frontotemporal dementia associated with C90RF72 mutations. Brain 2012;135:693-708.

-22 Chio A, Borghero G, Restagno G, et al: Clinical characteristics of patients with familial amyotrophic lateral sclerosis carrying the pathogenic GGGGCC hexanucleotide repeat expansion of C90RF72. Brain 2012;135:784-793.

23 Millecamps S, Boillee S, Le Ber I, et al: Phenotype difference between ALS patients with expanded repeats in C90RF72 and patients with mutations in other ALS-related genes. J Med Genet 2012;49:258-263.

24 Brettschneider J, Van Deerlin VM, Robinson JL, Kwong L, Lee EB, Ali YO, Safren N, Monteiro MJ, Toledo JB, Elman L, McCluskey L, Irwin DJ, Grossman M, Molina-Porcel L, Lee VM, Trojanowski JQ: Pattern of ubiquilin pathology in ALS and FTLD indicates presence of C90RF72 hexanucleotide expansion. Acta Neuropathol 2012;123:825-839.

-25 Stewart H, Rutherford NJ, Briemberg H, Krieger C, Cashman N, Fabros M, Baker M, Fok A, Dejesus-Hernandez M, Eisen A, Rademakers R, Mackenzie IR: Clinical and pathological features of amyotrophic lateral sclerosis caused by mutation in the C9ORF72 gene on chromosome 9p. Acta Neuropathol 2012;123:409-417.

-26 Byrne S, Elamin M, Bede P, et al: Cognitive and clinical characteristics of patients with amyotrophic lateral sclerosis carrying a C9orf72 repeat expansion: a population-based cohort study. Lancet Neurol 2012;11:232-240.

$\checkmark 27$ Cooper-Knock J, Hewitt C, Highley JR, et al: Clinico-pathological features in amyotrophic lateral sclerosis with expansions in C90RF72. Brain 2012;135:751-764.

-28 Mahoney CJ, Downey LE, Ridgway GR, Beck J, Clegg S, Blair M, Finnegan S, Leung KK, Yeatman T, Golden H, Mead S, Rohrer JD, Fox NC, Warren JD: Longitudinal neuroimaging and neuropsychological profiles of frontotemporal dementia with C90RF72 expansions. Alzheimers Res Ther 2012;4:41.

29 Hsiung GY, Dejesus-Hernandez M, Feldman HH, et al: Clinical and pathological features of familial frontotemporal dementia caused by C9ORF72 mutation on chromosome 9p. Brain 2012;135:709-722.

$\$ 30$ Whitwell JL, Weigand SD, Boeve BF, et al: Neuroimaging signatures of frontotemporal dementia genetics: C9ORF72, tau, progranulin and sporadics. Brain 2012;135:794-806.

31 Al-Sarraj S, King A, Troakes C, Smith B, Maekawa S, Bodi I, Rogelj B, Al-Chalabi A, Hortobagyi T, Shaw CE: p62 positive, TDP-43 negative, neuronal cytoplasmic and intranuclear inclusions in the cerebellum and hippocampus define the pathology of C9orf72-linked FTLD and MND/ALS. Acta Neuropathol 2011;122:691-702.

32 Agosta F, Vossel KA, Miller BL, Migliaccio R, Bonasera SJ, Filippi M, Boxer AL, Karydas A, Possin KL, GornoTempini ML: Apolipoprotein E epsilon4 is associated with disease-specific effects on brain atrophy in Alzheimer's disease and frontotemporal dementia. Proc Natl Acad Sci USA 2009;106:2018-2022.

-33 Vossel KA, Bien-Ly N, Bernardo A, Rascovsky K, Karydas A, Rabinovici GD, Sidhu M, Huang EJ, Miller BL, Huang Y, Seeley WW: ApoE and TDP-43 neuropathology in two siblings with familial FTLD-motor neuron disease. Neurocase 2013;19:295-301.

-34 Chow TW, Gao F, Links KA, Ween JE, Tang-Wai DF, Ramirez J, Scott CJ, Freedman M, Stuss DT, Black SE: Visual rating versus volumetry to detect frontotemporal dementia. Dement Geriatr Cogn Disord 2011;31:371-378.

35 Rossor MN, Fox NC, Mummery CJ, Schott JM, Warren JD: The diagnosis of young-onset dementia. Lancet Neurol 2010;9:793-806. 